\title{
Metabolic risk factors for liver inflammation in a cohort of chronic hepatitis $C$ patients
}

\author{
Mihaela Andreea Rădulescu ${ }^{1,2^{*}}$, Victoria Aramă ${ }^{1,2}$, Daniela loana Munteanu ${ }^{2}$, Raluca loana Mihăilescu2, \\ Cătălin Tilişcan ${ }^{1,2}$, Cristina Popescu ${ }^{1,2}$, Viorica Poghirc ${ }^{2}$, Irina Lăpădat ${ }^{2}$, Smaranda Gliga², Sorin Ștefan Aramă', \\ Adrian Streinu-Cercel ${ }^{1,2}$
}

From The 10th Edition of the Scientific Days of the National Institute for Infectious Diseases "Prof Dr Matei Bals"

Bucharest, Romania. 15-17 October 2014

\section{Background}

Beside the classic risk factors for disease progression in chronic hepatitis $\mathrm{C}(\mathrm{CHC})$ patients, metabolic disturbances came to attention in the last decade as important factors influencing disease progression and liver activity. This study aimed to evaluate metabolic factors in relation to liver inflammation in a cohort of $\mathrm{CHC}$ patients.

\section{Methods}

We conducted a cross-sectional non-interventional study on CHC patients evaluated in a tertiary hospital in Bucharest between December 2012-August 2013. We measured fasting serum lipids, glucose, liver transaminases, inflammatory proteins, and viral load. We calculated body-mass index (BMI) and waist-to-hip ratio (WTH). Cardiovascular risk was assessed with Framingham risk score, metabolic syndrome was defined with ATPIII criteria. Liver histology was assessed with noninvasive Fibromax tests (Biopredictive, France). For statistical analysis we used SPSS (version 12.0).

\section{Results}

We enrolled $117 \mathrm{CHC}$ patients compared to 30 uninfected controls. Median age was 54 years [45-61], sex ratio was F: M 1.8. Sixty-one patients (52.1\%) had low-grade activity score (A0-1) and 56 (47.8\%) had important liver activity (A2-3). In univariate analysis liver activity score (ActiScore) was correlated to age (Spearman rho $=0.353, \mathrm{p}<$ 0.001). ActiScore was correlated to FibroScore, SteatoScore and NashScore (Spearman rho $=0.770,0.528,0.439$,

\footnotetext{
* Correspondence: michelle_radulescu@yahoo.com

${ }^{1}$ Carol Davila University of Medicine and Pharmacy, Bucharest, Romania
}

Full list of author information is available at the end of the article $\mathrm{p}<0.001$ for all comparisons). Also ActiScore was correlated to viral load ( $r h o=0.262, \mathrm{p}=0.023$ ). Regarding metabolic factors, ActiScore was positively correlated to waist circumference and WTH ratio (rho $=0.200, \mathrm{p}=$ 0.034 , respectively rho $=0.290, \mathrm{p}=0.002$ ) and inversely correlated to serum cholesterol and LDL (rho $=-0.262$ and $-0.294, p=0.005$ for both). Also ActiScore was negatively correlated to $\mathrm{C}$-reactive protein and serum fibrinogen $($ rho $=-0.318, \mathrm{p}=0.001$ and rho $=-0.340, \mathrm{p}<0.001)$.

The number of criteria for metabolic syndrome and Framingham risk score were correlated to ActiScore (rho $=0.211, \mathrm{p}=0.034$, respectively rho $=0.510, \mathrm{p}<0.001)$. When we compared low activity (A0-1) to high activity (A2-3) patients in logistic regression, risk factors for high activity were age (OR 1.07), SteatoScore (OR 3.93) and NashScore (OR 3.79, p < 0.001 for all variables), while serum cholesterol, C-reactive protein and fibrinogen were protective factors (OR 0.042, 0.062, 0.071, p = $0.004,0.025$ and 0.008$)$.

\section{Conclusion}

In $\mathrm{CHC}$ patients' metabolic factors play an important role in disease activity. Liver metabolic disease had a negative predictive role, while serum cholesterol and inflammatory markers seemed to have a protective role against liver inflammation.

\footnotetext{
Authors' details

${ }^{1}$ Carol Davila University of Medicine and Pharmacy, Bucharest, Romania. ${ }^{2}$ National Institute for Infectious Diseases "Prof. Dr. Matei Balş", Bucharest, Romania.
}

Published: 15 October 2014 
doi:10.1186/1471-2334-14-57-014

Cite this article as: Rădulescu et al:: Metabolic risk factors for liver

inflammation in a cohort of chronic hepatitis C patients. BMC Infectious

Diseases 2014 14(Suppl 7):014.

Submit your next manuscript to BioMed Central and take full advantage of:

- Convenient online submission

- Thorough peer review

- No space constraints or color figure charges

- Immediate publication on acceptance

- Inclusion in PubMed, CAS, Scopus and Google Scholar

- Research which is freely available for redistribution

Submit your manuscript at 